\title{
Rapid cVEMP and oVEMP Responses Elicited by a Novel Head Striker and Recording Device
}

\author{
$* \dagger$ P. Ashley Wackym, *†Jennifer A. Ratigan, *Jonathan D. Birck, \\ *Steven H. Johnson, $\dagger$ Josef Doornink, †Michael Bottlang, † Stuart K. Gardiner, \\ and $*$ F. Owen Black
}

*Ear and Skull Base Center, and †Legacy Research Institute, Portland, Oregon, U.S.A.

\begin{abstract}
Objective: To develop a reliable, easy to use bedside, office, or field system that allows the rapid measurement of cervical and ocular vestibular evoked myogenic potentials (cVEMP and oVEMP) using a bone-conduction stimulus.

Study Design: Prospective bioengineering design and proof of concept of the test system with saccular and utricular otolith response studies in human subjects.

Setting: Private practice, tertiary neurotology referral center. Subjects: Twenty healthy adult controls without history of auditory or vestibular dysfunction and 5 preoperative and postoperative patients with confirmed superior canal dehiscence (SCD) participated.

Interventions: The subjects underwent auditory stimuli-based cVEMP and oVEMP studies using a commercially available system as well as testing with a novel bone-conduction cVEMP and oVEMP head striker system.

Main Outcome Measures: Duration of each study, healthy subject and patient comfort, reproducibility, latency, and amplitude of auditory and striker evoked cVEMP and oVEMP responses. Results: The mean age of the healthy controls was 43.8 , with a range of 19 to 69 years (10 male and 10 female subjects). The mean age of the SCD patient group was 46 , with a range of 25 to 54 years; all female subjects. Although the cVEMP responses
\end{abstract}

were similar using either the auditory or head strike stimuli, the healthy subjects preferred the latter, but the SCD patients became more symptomatic. The oVEMP data showed more consistent responses using the striker system. A statistically significant reduction in latency for the striker-evoked cVEMP occurred compared with the auditory cVEMP evoked response in the 5 SCD preoperative patients. All normalized postoperatively.

Conclusion: Recording the cVEMP and oVEMP responses using the striker system was much more rapid than with auditory stimuli and was more comfortable for the healthy subjects. The striker system and the acoustic method elicited strong otolithic receptor dysfunction symptoms in all SCD patients; however, they preferred the shorter striker studies. The striker system, because of the statistically shorter latency of p13 during the striker evoked cVEMP, which normalized after SCD closure, suggests that this method may be useful in identifying SCD patients before imaging studies. In addition, based on our biomechanical data, the striker was able to reliably produce a consistent and defined head striker impact. Key Words: Cervical-DizzinessEndolymphatic hydrops-Latency-Ocular-Superior canal dehiscence-Vestibular evoked myogenic potentials.

Otol Neurotol 33:1392-1400, 2012.
It was over a century ago that Robert Bárány began using caloric irrigation and his vertical axis rotational chair to assess horizontal canal function, yet it was not until 1994 that Colebatch and colleagues developed the soundevoked cervical vestibular evoked myogenic potential (cVEMP) (1) to study the gravitational receptors. Soundinduced activation of the saccule leads to an inhibition of the sternocleidomastoid muscle (SCM), and this inhibi-

Address correspondence and reprint requests to P. Ashley Wackym, M.D., Ear and Skull Base Center, 1225 NE 2nd Avenue,Suite 305, Portland, OR 97232; E-mail: wackym@neurotology.org

Joint first author: P. Ashley Wackym, and Jennifer A. Ratigan.

The authors disclose no conflicts of interest. tory potential can be recorded as the cVEMP (review $[2,3])$. The evoked potentials recorded from a number of other muscles have been studied as well; however, it is the SCM that is most consistently used in research and clinical applications. It has also been shown that both an ipsilateral and contralateral cVEMP can be recorded from the SCM after ipsilateral stimulation $(3,4)$.

In 1995, the first report that bone-conducted stimuli produce cVEMP responses was published (5); however, it was not until 2005 that bone-conducted stimuli were found to evoke extraocular muscle electromyography potentials (6). This led to the development of the ocular VEMP (oVEMP) test. Many have contributed to understanding the oVEMP response, particularly Ian Curthoys' group (2). Both acoustic and bone-conduction stimuli activate the 
saccular and utricular otolithic receptors; however, the otolithic input to the sternocleidomastoid muscle is predominately from the saccular macula, whereas the otolithic input to the inferior oblique muscle is predominately from the utricular macula (2,3). Thus, quantitatively, cVEMP tests saccular function, whereas oVEMP tests utricular function. Another practical extension of these relationships is that the cVEMP reflects inferior vestibular nerve function, whereas the oVEMP reflects the superior vestibular nerve function.

The earliest reports of using a bone-conducted stimulus to generate a cVEMP response used a reflex hammer (5) or a Radioear B-71 bone conduction oscillator (Radioear, New Eagle, PA, USA) (7). Since that time, a wide range of stimuli have been used including tapping the forehead with a reflex hammer, and other electromechanical devices such as a modified mini-shaker (Mini-Shaker 4810; Brüel \& Kjær, Nærum, Denmark) or custom-fabricated devices (review $(2,8,9)$ ). John Carey's group studied the testretest reliability of cVEMP and oVEMP testing using a wide range of stimuli and age-related characteristics of these tests (8). Although the reflex hammer tapping of the forehead produces the most robust cVEMP and oVEMP responses (8), there is no mechanism to standardize and calibrate the stimulus. The use of bone-conducted stimuli is desirable to study patients with conductive hearing losses as well as to decrease the stimulus time needed for the computer averaging of the responses.

Because of the limitations of the methods to induce a bone-conduction cVEMP and oVEMP response that would be of adequate magnitude and repeatability, we developed a rapid, computer-controlled, calibrated, and hand-held device that can deliver up to 2 strikes per second and with much greater strike intensity and consistency (Fig. 1). We also developed an integrated oVEMP recording system that can be rapidly used with multiple subjects (Fig. 1). The system was also designed so that it is portable, can be administered much faster than acoustic cVEMP and oVEMP studies, and saturates the receptors so that interaural differences are more accurate and consistent (reduced test-retest variability).

Nguyen et al. (8) provided an excellent review of pathologic conditions that have been studied using VEMPs. One disorder that is especially interesting regarding cVEMPs is superior canal dehiscence (SCD). These patients experience vertigo, oscillopsia, and/or sound-induced disequilibrium (10). Clinically, there has been a wide range and variability of symptoms associated with SCD, consistent with a spectrum. Because cVEMP and oVEMP responses have been reported to change when recorded preoperatively and postoperatively, we included SCD patients in this study.

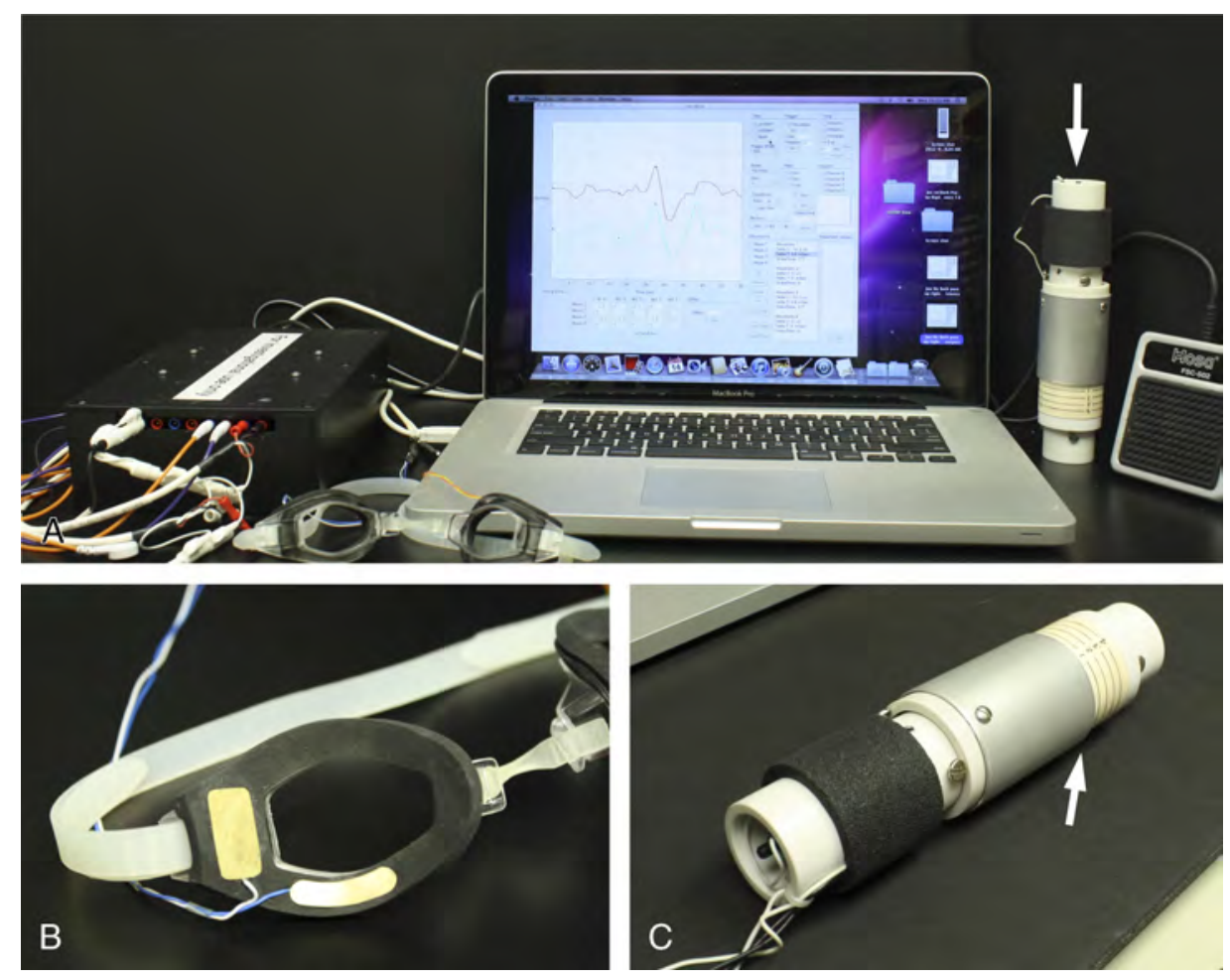

FIG. 1. Photographs of the striker cVEMP and oVEMP system $(A)$. The entire portable system, from left to right includes the computer interface, electrode goggles (foreground), laptop computer, hand-held striker (arrow), and foot petal trigger control (B). Detail of the electrodes built into the goggles for recording oVEMP responses $(C)$. The hand-held striker system has an outer sleeve (arrow) that is calibrated to allow uniform pressure, ranging from 1 to $4 \mathrm{lbs}$, of the end of the device (top right) to the patient's forehead at Fz. (Published with permission (c) 2012, Ear and Skull Base Center). 


\section{METHODS}

The procedures followed were in accordance with the ethical standards of the responsible committee on human experimentation and with the Helsinki Declaration. Our institutional review board approved these studies.

\section{Control Subjects}

Twenty healthy adults (10 male and 10 female subjects, with a mean age of 43.8 and a range of 19-69 yr; male mean age, 42.8 [range, 19-54]; and female mean age, 44.7 [range, 33-69]) with no hearing or vestibular deficits enrolled in the study. They were recruited from our institution, and all subjects were evaluated clinically including assessment of symptoms; however, a comprehensive auditory and vestibular diagnostic test battery was not performed.

\section{Patients}

Five SCD patients (all female, with a mean age of 46 and a range of 25-54 yr) were studied preoperatively and postoperatively. All 5 SCD patients underwent temporal craniotomy and plugging of their SCD.

All 25 subjects underwent both acoustic and bone-conduction (striker) stimuli studies to record cVEMP and oVEMP responses. Comparisons were made regarding latency, amplitude, duration of testing, and patient comfort.

\section{Acoustic cVEMP/oVEMP Stimuli and Recording Techniques}

A commercial auditory evoked potential system (Software version 6.2.1d, Bio-logic Systems Corp, Mundelein, IL, USA) was used for acoustic VEMP testing. Sound stimuli were delivered monaurally via intra-auricular transducer with foam E-A-R Link Inset Earphones (Aearo Company Auditory Systems, Indianapolis, IN, USA).

During the recording protocol, the subjects were seated upright. The skin, in areas of electrode placement, was cleansed with alcohol preps before electrode placement. cVEMP measurements were recorded using disposable, self-adhesive, pregelled, electrodes (3M Red Dot $\mathrm{Ag} / \mathrm{AgCl}$, London, Ontario, Canada) and lead wires from the Bio-logic Corp. The electrode montage consisted of an active electrode on the top third of the sternocleidomastoid muscle, a reference electrode on the sternoclavicular junction, and a ground electrode placed on the sternal notch. oVEMP measurements were recorded using disposable, selfadhesive, pregelled, electrodes (Ambu Neuroline 720, CareFusion, Middleton, WI, USA) with attached safety lead wires from Bio-logic Corp. The electrode montage consisted of an active electrode placed approximately $3 \mathrm{~mm}$ below the eye and centered beneath the pupil on the inferior ocular muscle, a reference electrode was placed approximately $2 \mathrm{~cm}$ laterally to the eye/medial temple, and a ground electrode remained on the sternal notch.

During the cVEMP instruction, patients were asked to rotate their head toward the contralateral shoulder from the stimulus, and tilt/angle approximately 30 degrees maximizing the contraction of the sternocleidomastoid muscle. The clinician applied the maximum amount of manual resistance that each subject could tolerate while visually confirming the SCM contraction during stimulus delivery. During the oVEMP instruction, patients were asked to maintain a maximum degree of gaze upward during stimulation after which a break was permitted.

During the cVEMP and oVEMP measurements, air conducted stimuli were delivered with $1000 \mathrm{~Hz}, 100 \mathrm{dBnHL}$ tone burst of positive polarity at a repetition rate of 4.3 per second (2-ms rise/ fall time, 2-ms plateau). Evoked myogenic potentials were amplified by $1000 \times$ and band-pass filtered $(10-1,500 \mathrm{~Hz})$. Average sweeps per test were approximately 80 to 200 , although the SCD patients were often unable to tolerate this many repetitions because they became symptomatic and were unable to maintain their gaze or head position.

The response parameters were defined as the cVEMP p13 potential being the first distinctive trough in the waveform, occurring approximately at the anticipated 10 to $14 \mathrm{~ms}$, poststimulus, and the $\mathrm{n} 23$ potential being the first distinctive peak in the waveform, occurring approximately 19 to $23 \mathrm{~ms}$ after stimulus

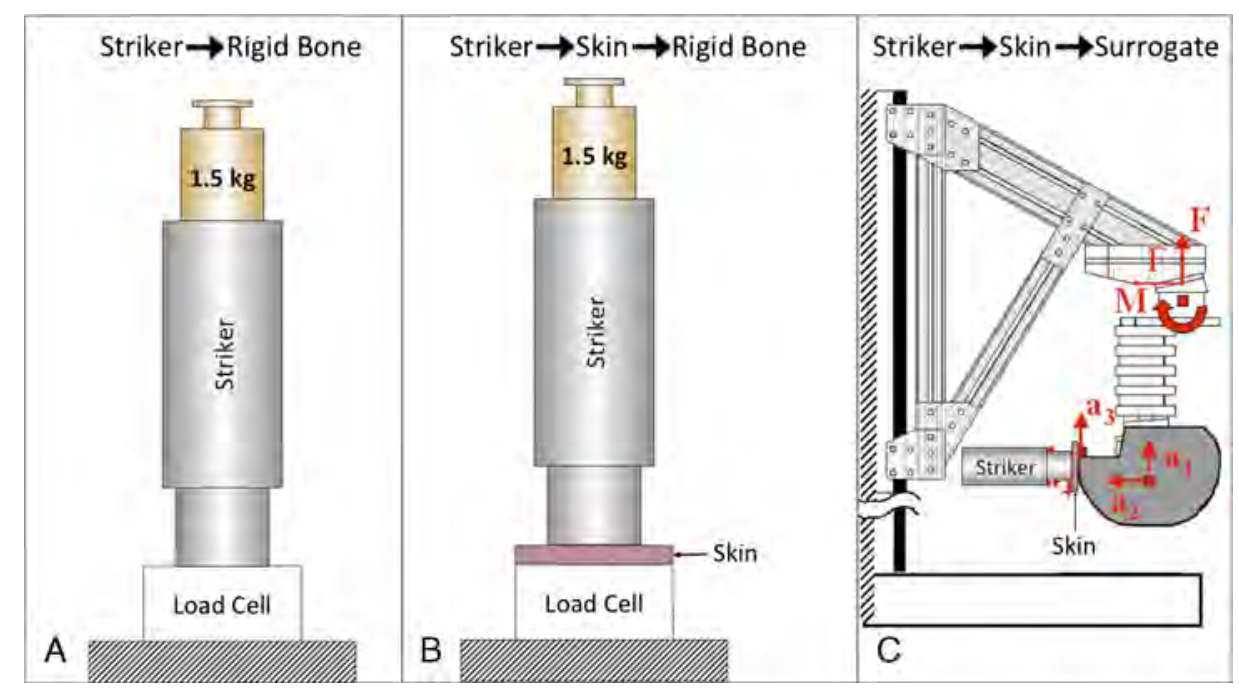

FIG. 2. The striker impact was evaluated under three conditions $(A)$. Direct impact on load cell $(B)$. Impact through fresh porcine skin $(C)$. Impact-induced acceleration of a head surrogate suspended on Hybrid III neck. Head acceleration was measured in terms of linear acceleration in anteroposterior direction $\left(\mathrm{a}_{2}\right)$, irrespective of neck moments $(\mathrm{M})$ and forces $(\mathrm{F})$. (Published with permission $\subset$ C 2012, Ear and Skull Base Center). 
TABLE 1. Normative data for acoustic-induced cervical and ocular vestibular evoked myogenic potentials

\begin{tabular}{|c|c|c|c|c|c|}
\hline & Parameter & Mean & SD & Range & $95 \% \mathrm{CI}$ \\
\hline \multirow[t]{5}{*}{ cVEMP } & p13 & 13.81 & 1.03 & $11.44-15.12$ & $13.26-14.36$ \\
\hline & $\mathrm{n} 23$ & 21.80 & 2.59 & $17.41-25.74$ & $20.42-23.18$ \\
\hline & ILD & 8.03 & 2.19 & $4.27-11.87$ & $6.86-9.19$ \\
\hline & $\mu \mathrm{V}$ & 53.34 & 44.05 & $11.44-178.09$ & $29.86-76.81$ \\
\hline & IAD & $32.51 \%$ & $24.62 \%$ & $1.86 \%-68.52 \%$ & $11.92 \%-53.09 \%$ \\
\hline \multirow[t]{5}{*}{ oVEMP } & n10 & 8.47 & 1.35 & $6.79-10.75$ & $7.68-9.25$ \\
\hline & p16 & 12.31 & 2.37 & $9.81-17.41$ & $10.94-13.68$ \\
\hline & ILD & 3.84 & 1.47 & $2.19-7.29$ & $2.99-4.69$ \\
\hline & $\mu \mathrm{V}$ & 5.46 & 3.55 & $1.52-14.34$ & $3.41-7.51$ \\
\hline & IAD & $32.03 \%$ & $25.23 \%$ & $0.00 \%-63.10 \%$ & $8.70 \%-55.37 \%$ \\
\hline
\end{tabular}

There were 20 healthy subjects (10 male and 10 female subjects) studied. Amplitude of the p13-n23 response and the n10-p16 response $(\mu \mathrm{V})$; latency of the p13 and $\mathrm{n} 23$ [cVEMP] and $\mathrm{n} 10$ and p16 [oVEMP] peaks are in milliseconds (ms).

CI indicates confidence interval; cVEMP, cervical vestibular evoked myogenic potential; IAD, interaural amplitude difference; ILD, interlatency difference in milliseconds; oVEMP, ocular vestibular evoked myogenic potential; SD, standard deviation.

onset. The oVEMP n10 potential was identified as the first distinctive and replicated peak in the waveform, poststimulus, at the anticipated 8 to $12 \mathrm{~ms}$. A p16 waveform was identified as the replicated trough following the peak p10 at approximately $16 \mathrm{~ms}$ poststimulus. Peak-to-peak amplitude was calculated using the Bio-logic software; after peaks were labeled and encompassing the amplitude difference between the 2 peaks. The asymmetry ratio between a subject's ears was calculated according to the following formula and expressed as a percentage.

$\operatorname{AR}($ asymmetry ratio $)=\frac{(\text { Left amplitude }- \text { Right amplitude })}{(\text { Left amplitude }+ \text { Right amplitude })} \times 100$

\section{Striker cVEMP/oVEMP Stimuli and Recording Techniques}

We developed a novel evoked potential and recording system that was used for the nonauditory cVEMP and oVEMP studies. Stimuli were delivered to each subject's midline forehead at Fz, while seated, via a handheld striker (Fig. 1). oVEMP measurements were recorded using electrode plates, designed and mounted to commercially available swim goggles, with attached lead wires from the experimental evoked potential system (Fig. 1). The electrode montage consisted of an active (negative) electrode mounted in the goggles, aligning below the eye overlying the inferior rectus and inferior oblique muscles; a reference (positive) electrode was placed on the lateral portion of the goggles, aligning with the lateral eye, and a ground electrode, via disposable, pregelled, self-adhesive (3M Red Dot $\mathrm{Ag} / \mathrm{AgCl}$ London, Ontario, Canada) placed on the sternum. The skin, in areas of the goggles, was cleansed with alcohol prep and saturated with conductivity gel (Lectron II, Pharmaceutical Innovations, Newark, NJ, USA) before electrode placement. The cVEMP measurements were recorded using the same electrode montage used for the acoustic cVEMP studies.

For the cVEMP instructions, the subjects were told to attempt a head turn toward the recorded side, against hand-pushed resistance, maximizing the contraction of the sternocleidomastoid muscle. For the oVEMP instructions, the subjects were told to maintain a maximum degree of gaze upward during stimulation. Duration lasted 8 seconds per recording, and 2 recordings were obtained per side.
For the cVEMP and oVEMP measurements, stimuli were delivered via 16 strikes to the midline forehead at the hairline $(\mathrm{Fz})$ at a rate of $2 /$ second, and the responses were averaged. No input filters were used on the acquisition system signals, thereby leaving the signal amplitude and phase undisturbed.

The cVEMP and oVEMP response parameters, peak-to-peak amplitudes, ILD and IAD (asymmetry ratio), were defined and calculated as described earlier; however, the striker system software was used to perform these calculations.

\section{Acoustic and Striker cVEMP and oVEMP Statistical Analysis}

Generalized estimating equation models were formed to determine the effect of acoustic or striker method on each parameter in turn, for each of cVEMP and oVEMP responses, within the control subjects. This method accounts for the fact that there were 2 measurements per individual, on the left and right sides. Next, ordinary least-squares linear regression models were formed to determine the effect of method on the postoperative SCD parameter values, for the treated and untreated sides separately. Finally, the parameter values using the striker method were compared preoperatively and postoperatively for the SCD patients. In each case, the distribution of residuals was plotted and approximately Gaussian, enabling these parametric analyses to be valid. For statistical purposes, the interaural amplitude difference (IAD) was used as a positive value, thereby removing right-left differences.

\section{Striker Force Validation}

The direct impact of the striker was measured using a compression load sensor (1 kN capacity, Model 2527-620, Instron, Canton, MS, USA) and the data acquisition system of a calibrated material test station (8841; Instron) (Fig. 2). Three sets of experiments were designed to characterize the striker impact. For all of these tests, 3 repeat impacts were performed, and the resulting impact and acceleration results were averaged.

To quantify the striker impact when placed in direct contact to a rigid load cell, the striker was placed in direct contact with the load sensor (Fig. 2A). A static weight of $1.5 \mathrm{~kg}$ was used to compress the striker onto the load sensor with a static preload of $14.7 \mathrm{~N}$ (9). While maintaining this static preload, the impact force generated by the striker was recorded at a sample rate of $5 \mathrm{kHz}$ with the $1 \mathrm{kN}$ load cell. The peak impact force and the duration of the impact were extracted from the recoded data.

TABLE 2. Normative data for striker-induced bone conduction cervical and ocular vestibular evoked myogenic potentials

\begin{tabular}{|c|c|c|c|c|c|}
\hline & Parameter & Mean & SD & Range & $95 \% \mathrm{CI}$ \\
\hline \multirow[t]{5}{*}{ cVEMP } & p13 & 13.58 & 1.43 & $10.90-17.80$ & 13.12 \\
\hline & $\mathrm{n} 23$ & 20.37 & 3.46 & $15.70-$ & 19.2 \\
\hline & ILD & 6.79 & 3.07 & $2.50-19.30$ & 7.77 \\
\hline & $\mu \mathrm{V}$ & 63.14 & 34.70 & 10.30 & 52.04 \\
\hline & IAD & $15.58 \%$ & $11.05 \%$ & $0.78 \%-36.47 \%$ & $10.41 \%-20.76 \%$ \\
\hline \multirow[t]{5}{*}{ oVEMP } & $\mathrm{n} 10$ & 8.46 & 1.06 & $5.20-11.40$ & $8.12-8.80$ \\
\hline & p16 & 11.75 & 1.55 & $7.90-15.60$ & $11.25-12.25$ \\
\hline & ILD & 3.29 & 0.97 & $1.70-$ & $2.98-3.60$ \\
\hline & $\mu \mathrm{V}$ & 13.85 & 9.39 & $3.00-34.60$ & $10.85-16.85$ \\
\hline & IAD & $29.35 \%$ & $24.85 \%$ & $0.48 \%-84.00 \%$ & $17.71 \%-40.98 \%$ \\
\hline
\end{tabular}

There were 20 healthy subjects (10 male and 10 female subjects) studied. Amplitude of the p13-n23 response and the n10-p16 response $(\mu \mathrm{V})$; latency of the $\mathrm{p} 13$ and $\mathrm{n} 23$ [cVEMP] and $\mathrm{n} 10$ and $\mathrm{p} 16$ [oVEMP] peaks are in milliseconds $(\mathrm{ms})$. 

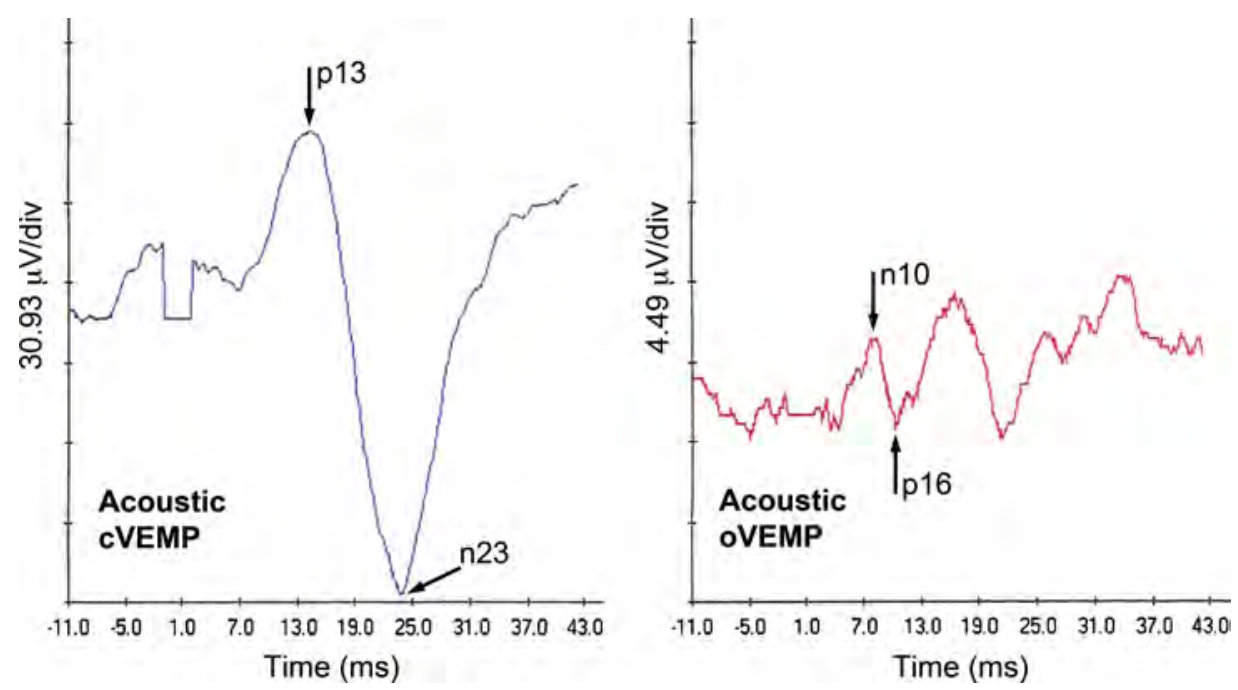

FIG. 3. Acoustic cVEMP (left) and oVEMP (right) from a healthy control subject. (Published with permission (C) 2012, Ear and Skull Base Center).

To quantify the striker impact delivered through skin and to determine the amount of impact energy absorbed by skin, a fresh porcine skin layer $(10 \times 10 \mathrm{~cm}[3-\mathrm{mm}$ thickness], [11]) was placed between the striker and the load sensor to simulate impulse absorption in vivo as the striker is compressed onto a patient's forehead (Fig. 2B). While maintaining a constant preload of $14.7 \mathrm{~N}$, the impact generated by the striker that penetrated through the skin layer onto the load cell was recorded. The amount of impact absorbed by the skin layer was calculated by subtracting the impact load measured with the skin layer from the impact load measured without the skin layer. Statistical analysis on the effect of the porcine skin layer on impact absorption was performed using 2-tailed, unpaired Student's $t$ tests at a level of significance of $\alpha=0.05$.

To quantify the head acceleration caused by the striker, a head and neck surrogate (International Organization for Standardization [ISO] headform, Hybrid III neck) was used representing a reproducible and clinically relevant test scenario (Fig. 2C). The striker was placed on the Fz of a head and neck surrogate. This surrogate represents the mass and mass distribution of a medium-sized head, whereby the neck surrogate provides constraints that ensure biofidelic head kinematics upon impact with the striker. Head acceleration (front-to-back) was measured with an acceleration sensor (Model 356B21; PCB Piezotronics, Depew, NY, USA) mounted at the center of gravity of the head surrogate. The same porcine skin layer was placed between the striker and the head surrogate. Using the hand-held compression scale incorporated into the striker, a static preload of approximately 14.7 $\mathrm{N}$ was manually applied to compress the striker onto the skin. The signal was filtered in accordance with Society of Automotive Engineers standards (SAE J211), and the head acceleration peak value and duration in response to a striker impact was recorded.

\section{RESULTS}

Tables 1 and 4 summarize the findings of the cVEMP and oVEMP studies of human subjects. Although the cVEMP responses were similar using either the auditory or striker (nonauditory) stimuli, the healthy subjects preferred the striker because of the rapidity of the test and the avoidance of the perceived loud auditory stimulus. Tables 1 and 2 summarize the normative data obtained from our healthy subjects. The cVEMP and oVEMP responses recorded in our healthy subjects using acoustic stimulation were comparable to other published studies (Fig. 3) $(8,12)$; however, our striker oVEMP response amplitudes were significantly greater than with acoustic stimulation (Table 3 ). We also observed a significant reduction in the cVEMP IAD in our healthy subjects using the striker compared with acoustic stimulation (Table 3). The striker cVEMP and oVEMP responses were reproducible with appropriate latencies (Fig. 4). This was also true for the trials of bilateral simultaneous cVEMP and oVEMP recordings in response to the striker, although some of the healthy subjects had difficulty completing this task, none of the SCD patients could complete these studies (Fig. 5). The data reported herein are all from unilateral recordings.

For the oVEMP studies, the goggles were well tolerated, and they reduced the time needed for the audiologist

TABLE 3. Comparison of mean acoustic and striker vestibular evoked myogenic potentials in healthy subjects

\begin{tabular}{lcccc}
\hline & Parameter & Acoustic & Striker & $p$ \\
\hline cVEMP & p13 & 13.81 & 13.58 & 0.4926 \\
& n23 & 21.80 & 20.37 & 0.0844 \\
& ILD & 8.03 & 6.79 & 0.0837 \\
& $\mu V$ & 53.34 & 63.16 & 0.4122 \\
oVEMP & IAD & $32.51 \%$ & $15.59 \%$ & $0.0172^{a}$ \\
& n10 & 8.47 & 8.46 & 0.9830 \\
& p16 & 12.31 & 11.75 & 0.3934 \\
& ILD & 3.84 & 3.29 & 0.1810 \\
& $\mu V$ & 5.46 & 13.85 & $<0.0001^{a}$ \\
& IAD & $32.03 \%$ & $29.34 \%$ & 0.8083
\end{tabular}

${ }^{a}$ Statistically significant $(p<0.05)$. There were 20 healthy subjects (10 male and 10 female subjects) studied. Amplitude of the p13-n23 response and the n10-p16 response $(\mu \mathrm{V})$; latency of the p13 and $n 23$ [cVEMP] and n10 and p16 [oVEMP] peaks are in milliseconds (ms). 

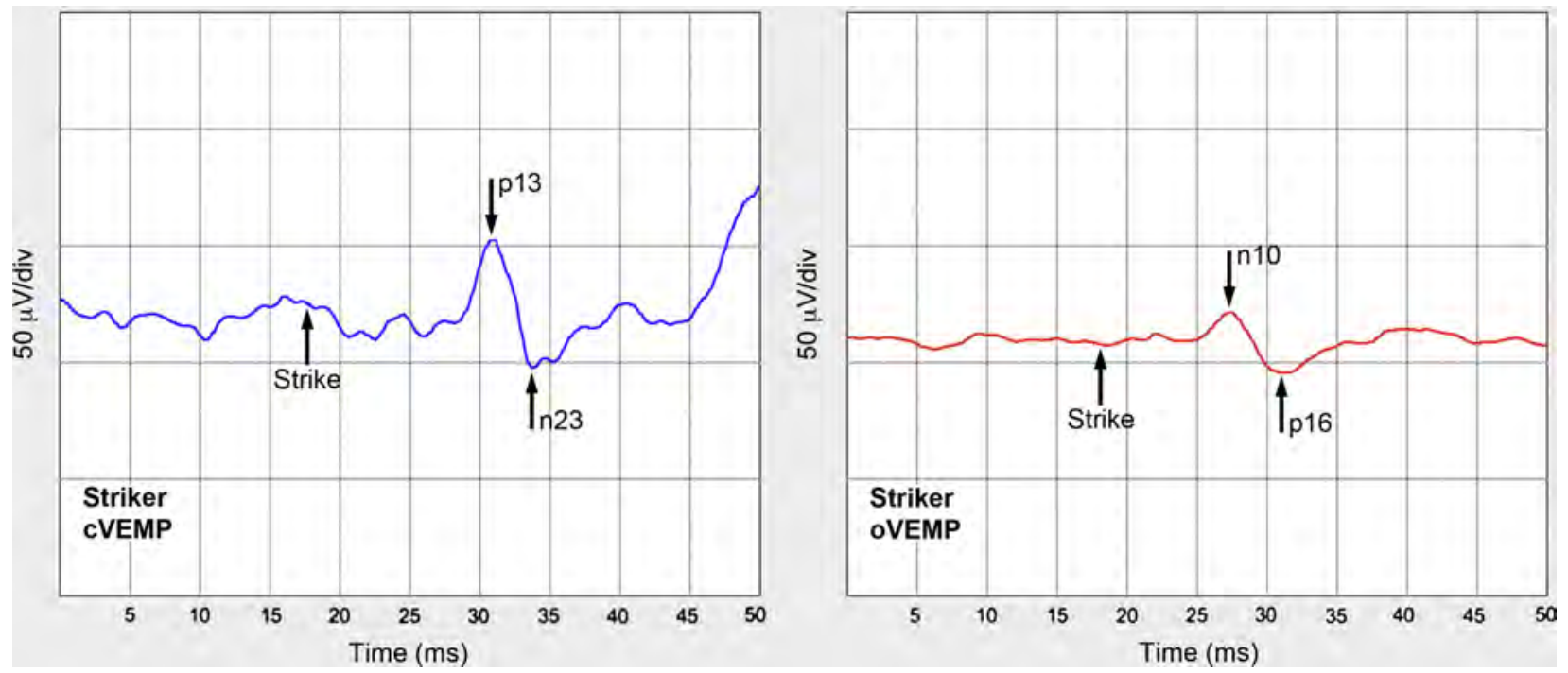

FIG. 4. Striker cVEMP (left) and oVEMP (right) from the same healthy control subject shown in Figure 3. Note the robust evoked potentials with appropriate location of $\mathrm{p} 13$ and $\mathrm{n} 23$ (cVEMP) and $\mathrm{n} 10$ and p16 (oVEMP). The striker system software records the time of the initial strike and the time to the initial peak and then calculates the $\Delta t$, which, in this example, was $13.2 \mathrm{~ms}$ (cVEMP) and 9.6 ms (oVEMP). This early version of the software did not automatically zero out the strike time; therefore, the time scale values shown at the bottom of the figure while accurate, do not represent the actual latency. (Published with permission (c) 2012, Ear and Skull Base Center).

to manage the electrodes typically used to record oVEMP responses.

Although the SCD patients experienced otolithic symptoms with acoustic stimulation, they became even more symptomatic with the striker stimulation; however, they preferred the striker because the duration of stimulation was much shorter. The oVEMP data showed more consistent responses using the striker system. A statistically significant reduction in latency for the striker cVEMP compared with the auditory cVEMP response in the 5 SCD preoperative patients, which normalized postoperatively (Table 4; Fig. 6). This was true for both the ipsilateral and contralateral sides.

\section{Striker Force Validation}

The peak impact force of the striker was reduced 54\% when impacting the porcine skin $(109.4 \pm 5.8 \mathrm{~N})$ compared with direct contact with the load cell $(237.3 \pm 3.3 \mathrm{~N}$, $p<0.001$; Fig. 7]; Table 5). The impulse duration increased $89 \%$ when impacting the porcine skin $(1.7 \mathrm{~ms})$ compared with direct contact with the load cell $(0.9 \mathrm{~ms})$.

Using the headform, the maximum front-to-back acceleration produced by the striker was $1.8 \pm 0.3 \mathrm{~g}$, and impulse of duration was $1.7 \pm 0.1 \mathrm{~ms}$.

\section{DISCUSSION}

Although this is a preliminary report, 2 statistically significant differences were observed between the acoustic versus striker stimuli that warrant discussion (Table 4). First, there was much less cVEMP IAD in healthy subjects when the striker was used; $15.59 \%$ with the striker compared with $32.51 \%$ with acoustic stimulation $(p=0.0172)$. It is possible that in the healthy controls, the intensity of the striker bone-conduction stimulation approaches saturation of the saccule and produces more accurate, symmetric responses. Second, the mean amplitude of the striker

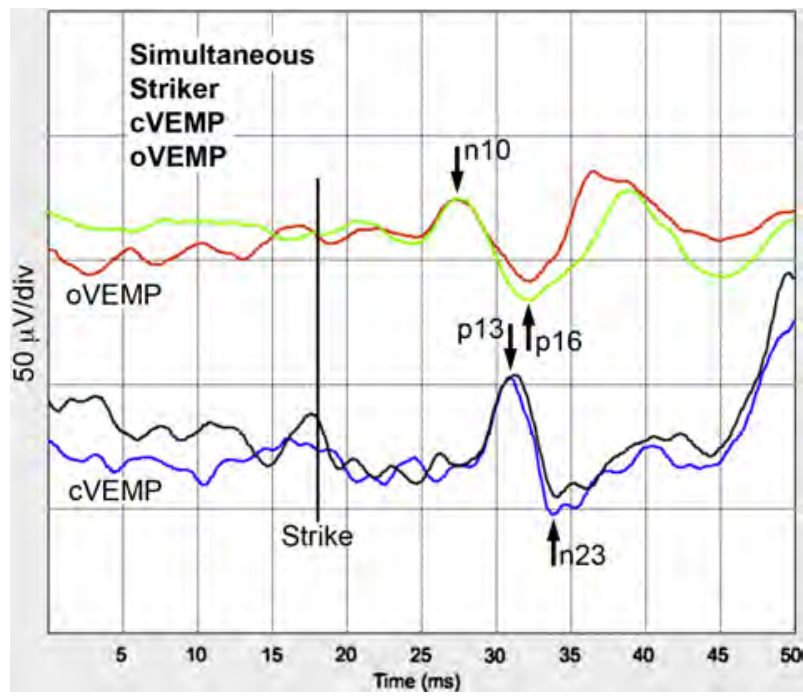

FIG. 5. Simultaneous oVEMP and cVEMP recorded from a healthy subject using the striker system. Note the waveform replication and expected cVEMP and oVEMP latencies. These responses were generated with 1 single 8-second stimulus intervals. The waveforms represent oVEMP right (red) and left (green), and cVEMP right (black) and left (blue). The striker system software records the time of the initial strike and the time to the initial peak and then calculates the $\Delta t$, which, in this example, was $9.5 \mathrm{~ms}$ (red oVEMP plot) and $9.9 \mathrm{~ms}$ (green OVEMP plot), and $13.2 \mathrm{~ms}$ (blue cVEMP plot) and $13.7 \mathrm{~ms}$ (black cVEMP plot). This early version of the software did not automatically zero out the strike time; therefore, the time scale values shown at the bottom of the figure, although accurate, do not represent the actual latency. (Published with permission (c) 2012, Ear and Skull Base Center). 
TABLE 4. Striker-induced vestibular evoked myogenic potentials, preoperative and postoperative repair of superior canal dehiscence

\begin{tabular}{|c|c|c|c|c|c|c|c|}
\hline & \multirow[b]{2}{*}{ Parameter } & \multicolumn{3}{|c|}{ Right side (superior canal dehiscence side) } & \multicolumn{3}{|c|}{ Left side } \\
\hline & & Preoperative & Postoperative & $p$ & Preoperative & Postoperative & $p$ \\
\hline \multirow[t]{4}{*}{ cVEMP } & p13 & 5.82 & 12.86 & $0.0003^{a}$ & 5.68 & 13.00 & $0.0012^{a}$ \\
\hline & $\mathrm{n} 23$ & 16.16 & 18.52 & 0.1456 & 15.16 & 18.76 & 0.1047 \\
\hline & ILD & 10.34 & 5.30 & $0.0075^{a}$ & 9.48 & 5.76 & 0.0681 \\
\hline & $\mu \mathrm{V}$ & 87.18 & 73.74 & 0.4311 & 129.80 & 67.84 & 0.2916 \\
\hline \multirow{4}{*}{ oVEMP } & n10 & 8.26 & 8.80 & 0.4101 & 8.121 & 8.23 & 0.9157 \\
\hline & p16 & 12.14 & 11.95 & 0.8989 & 11.62 & 10.97 & 0.6231 \\
\hline & ILD & 3.88 & 3.52 & 0.6811 & 3.50 & 2.75 & 0.0742 \\
\hline & $\mu \mathrm{V}$ & 38.92 & 13.72 & $0.2088^{b}$ & 30.10 & 13.00 & $0.3178^{b}$ \\
\hline
\end{tabular}

There were 5 female superior canal dehiscence subjects studied. Amplitude of the p13-n23 response and the n10-p16 response ( $\mu \mathrm{V})$; latency of the p13 and 23 [cVEMP] and n10 and p16 [oVEMP] peaks are in milliseconds (ms).

${ }^{a}$ Statistically significant $(p<0.05)$.

${ }^{b}$ The change in amplitude of the n10-p16 response was not statistically significant due to variability and the small number of averaged responses.

oVEMP was greater compared with the mean acoustic oVEMP, and this was highly significant $(p<0.0001)$. Following the study of a much larger cohort of healthy subjects and a wide array of patients with vestibular dysfunction, this may prove to yield improved diagnostic information regarding utricular function. A similar difference in cVEMP and oVEMP amplitude was observed when comparing acoustic stimuli (tones or clicks) with boneconduction stimuli (reflex hammer or minishaker) (8). To confirm these initial observations, future studies with a much larger and age stratified study population together with a study design that incorporates test-retest reliability (8) are necessary.

The goggles were well tolerated and recorded appropriate oVEMP responses. They provide uniform pressure around the eyes and consistent electrode placement. They would also be useful in studies conducted during parabolic flights and for oVEMP studies in the field or

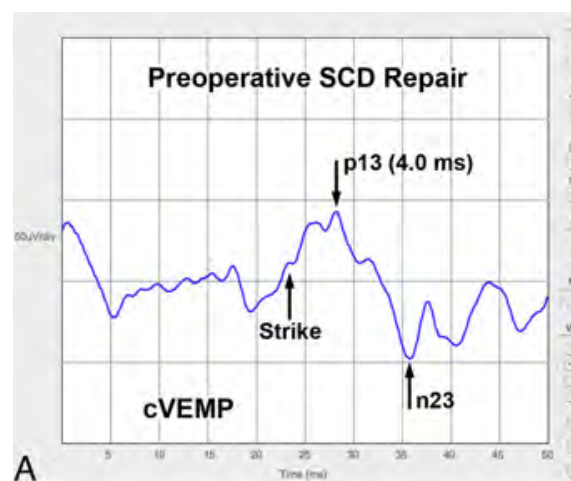

A

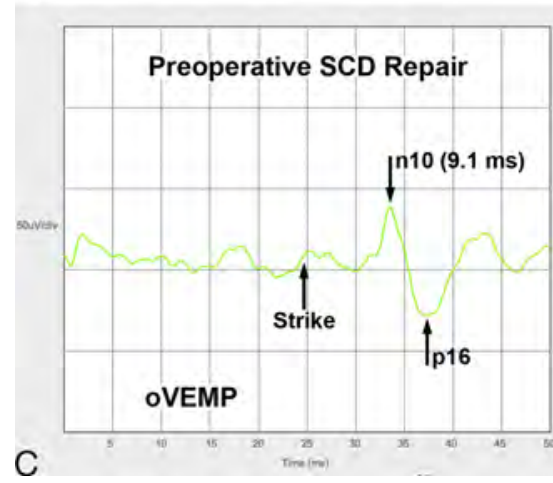

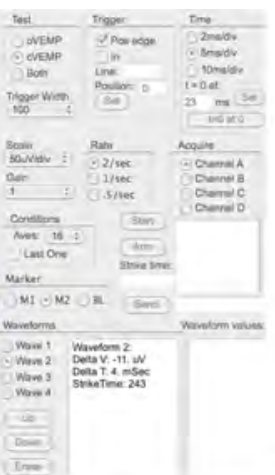

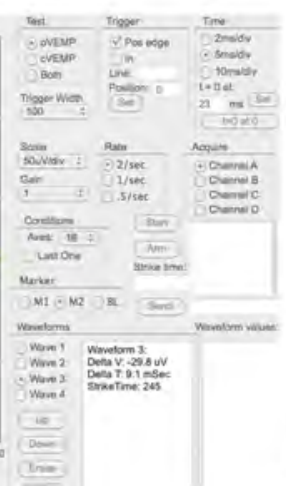

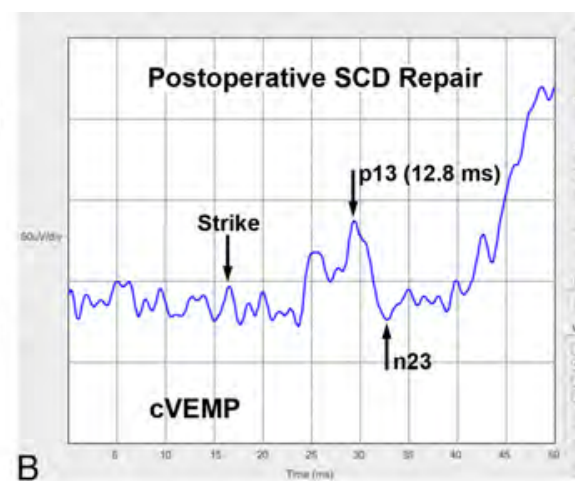
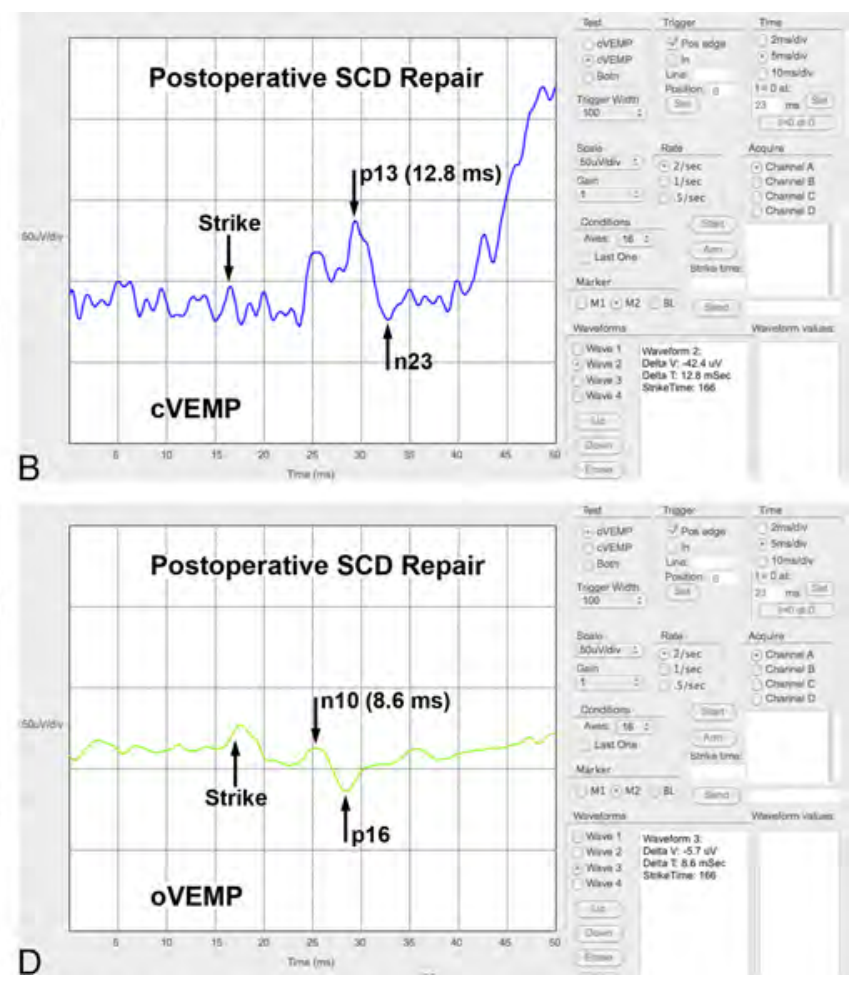

FIG. 6. Preoperative and postoperative SCD repair cVEMP and oVEMP responses. The striker system software records the time of the initial strike and the time to the initial peak and then calculates the $\Delta t$, which is shown in each panel (bottom right), and each of these time points are indicated (arrows). This early version of the software did not automatically zero out the strike time; therefore, the time scale values shown at the bottom of the figure, although accurate, do not represent the actual latency $(A)$. The latency of the preoperative cVEMP p13 is extremely short $(B)$. Postoperatively, the cVEMP p13 latency normalizes $(C)$. There is no change in the latency of the oVEMP n10 preoperatively $(D)$. The latency of the oVEMP n10 does not change postoperatively. Note the increased oVEMP amplitude preoperatively that normalizes postoperatively. (Published with permission ㄷ 2012, Ear and Skull Base Center). 

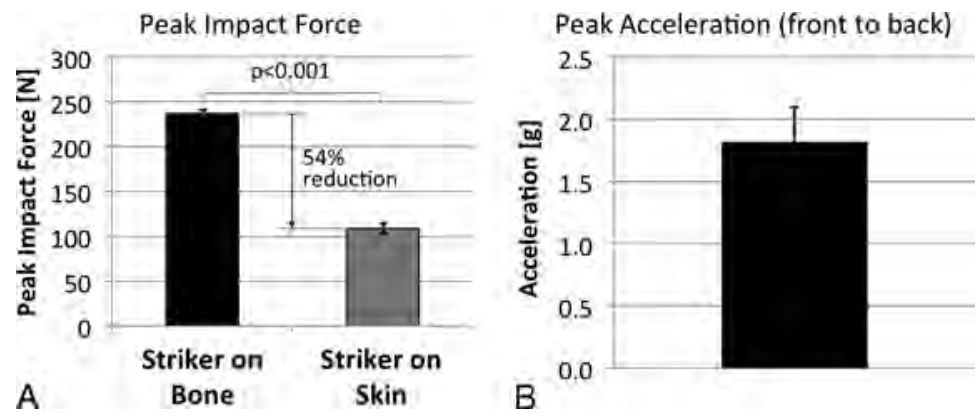

FIG. 7. Peak force and acceleration measured while delivering striker stimuli $(A)$. The striker impact on a rigid load cell shows that skin reduces the force transmitted to the bone by $54 \%(B)$. Peak acceleration of a validated headform/neck surrogate with the striker placed against skin to simulate clinical application. (Published with permission (c) 2012, Ear and Skull Base Center).

bedside, particularly if an audiologist or neurotologist were not placing the electrodes.

The striker stimulus was much more rapid than acoustic stimulus. For the striker system, 8 seconds of stimulus once for simultaneous bilateral cVEMP and oVEMP or 8 seconds twice for simultaneous unilateral cVEMP and oVEMP followed by simultaneous unilateral cVEMP and oVEMP of the other side. The striker system has the potential for performing bilateral simultaneous cVEMP and oVEMP studies; however, although simultaneous cVEMP and oVEMP recording is merely difficult for the control subjects, it was not possible for the SCD patients to tolerate. The acoustic stimuli for each of the 4 measurements, cVEMP and oVEMP for both the right and left side, varied between 30 seconds to 2 minutes (time for collection of 60-200 accepted averages). There was additional time added between each of the 4 tests for the patient to have a break. This was especially problematic for the SCD patients as each stimulus resulted in increased symptoms of otolithic dysfunction. The SCD patients preferred the two 8-second stimuli with the striker to the longer acoustic cVEMP and oVEMP studies. The shorter duration of testing may also be useful in other populations who are challenging to test; children and patients who have difficulty maintaining tonic contraction of the SCM are 2 such examples. The major negative aspect of the short stimulus time is that less averaging can be completed. Although we were able to record simultaneous bilateral cVEMP and oVEMP responses (Fig. 5), they were not tolerated by any of the SCD patients. In addition, there are physiologic reasons not to attempt bilateral simultaneous recordings $(2,3)$.

There is an important diagnostic role of measuring cVEMP responses in patients with SCD (review $[3,8]$ ). The third window creates a low-impedance pathway for energy to be transmitted to the labyrinth. Interestingly, with our striker-induced cVEMP responses, there was a statistically significant reduction in the p13 latency (Table 4; Fig. 6). This reduction was seen bilaterally despite the SCD being present in only the right side in all 5 patients. It is likely that the observed shorter latency on the non-SCD side is due to the contralateral response from an ipsilateral stimulation $(2,3)$. With the marked increase in force delivered via our device (Table 5), we hypothesize that a much more efficient and forceful stimulation of the saccule on the SCD side resulted in the shorter latency with less time required to activate the saccule. This reduced latency normalized in all of the patients after surgical closure of the SCD via a right temporal craniotomy and plugging technique. Increased p13 and n23 latencies have been reported in patients with central vestibulopathy (review [3]). We also hypothesize that there was no change in the oVEMP latencies because the utricle is not in direct contact with the bony labyrinth as the saccule is in the fossa ovalis. Confirmation of these phenomena in a much larger cohort of SCD patients and also with varying sizes of dehiscence is essential to determine if there is any potential diagnostic role of this system in these patients. Some centers will decrease the presentation level of the auditory stimulus in SCD patients to determine the threshold of the cVEMP response; however, many centers do not do so. With the striker stimulus used in our system, there is only one stimulation force; therefore, there is no capacity to determine the threshold necessary to evoke a response. Finally, the mean oVEMP amplitude was greater in the SCD patients but was not statistically significant in our small sample size.

Based upon our biomechanical studies, the striker system provides more consistent, calibrated, and intense stimuli than any other bone-conducting method/system developed since the first introduction of bone-conducted stimuli in 1995 (3-9) (Table 5). Stimulation from the striker was reliable and resulted in less than 3\% variation between strikes. Striker load was more than 7 times that measured by Brantberg et al. (9), but the use of the striker at this load and reported herein have been tolerated clinically. Peak acceleration was 9 times higher than that in the study by Brantberg et al.; however, the impact force in this study was higher than that in their study (Table 5). Additionally, measurements were made using a head neck/surrogate that is designed for impacts at a greater velocity. The neck of this surrogate has not been tested for

TABLE 5. Experimental peak impact force and peak acceleration compared to published data

\begin{tabular}{lcc}
\hline & Current study & Brantberg et al. (9) \\
\hline Peak impact force $(\mathrm{N})$ & 237.3 & 33 \\
Peak acceleration $(\mathrm{g})$ & 1.8 & 0.2 \\
\hline
\end{tabular}


biofidelity in these loading conditions, and it is likely more ridged than a realistic patient response. Systems currently designed to measure head impacts in sports have a minimum acceleration threshold of $10 \mathrm{~g}$ (13). This is 5 times the acceleration observed in this study. Based on these data, the striker was able to reliably produce a consistent and defined skull impact.

\section{CONCLUSION}

Recording the cVEMP and oVEMP responses using the striker system was much more rapid than with auditory stimuli and was more comfortable for the healthy subjects. The striker system and the acoustic method elicited strong otolithic receptor dysfunction symptoms in all SCD patients; however, they preferred the shorter striker studies. The striker system, because of the statistically shorter latency of p13 during the striker evoked cVEMP, which normalized after SCD closure, suggests that this method may be useful in identifying SCD patients before imaging studies. In addition, based on our biomechanical data, the striker was able to reliably produce a consistent and defined head striker impact.

Acknowledgments: The authors thank Ian Curthoys, Ph.D., for insightful discussion of our findings and constructive suggestions after reviewing the manuscript.

\section{REFERENCES}

1. Colebatch JG, Halmagyi GM, Skuse NF. Myogenic potentials generated by click-evoked vestibulocollic reflex. J Neurol Neurosurg Psychiatry 1994;57:190-7.
2. Curthoys IS. A critical review of the neurophysiological evidence underlying clinical vestibular testing using sound, vibration and galvanic stimuli. Clin Neurophysiol 2010;121:132-44.

3. Welgampola MS, Colebatch JG. Characteristics and clinical applications of vestibular-evoked myogenic potentials. Neurology 2005; 64:1682-8.

4. McNerney KM, Burkard RF. The vestibular evoked myogenic potential (VEMP): air- versus bone-conducted stimuli. Ear Hear 2011; 32:e6-e15.

5. Halmagyi GM, Yavor RA, Colebatch JG. Tapping the head activates the vestibular system: a new use for the clinical reflex hammer. Neurology 1995;45:1927-9.

6. Rosengren SM, McAngus Todd NP, Colebatch JG. Vestibularevoked extraocular potentials produced by stimulation with boneconducted sound. Clin Neurophysiol 2005;116:1938-48.

7. Welgampola MS, Rosengren SM, Halmagyi GM, Colebatch JG. Vestibular activation by bone conducted sound. J Neurol Neurosurg Psychiatry 2003;74:771-8.

8. Nguyen KD, Welgampola MS, Carey JP. Test-retest reliability and age-related characteristics of the ocular and cervical vestibular evoked myogenic potential tests. Otol Neurotol 2010;31:793-802.

9. Brantberg K, Löfqvist L, Westin M, Tribukait A. Skull tap induced vestibular evoked myogenic potentials: an ipsilateral vibration response and a bilateral head acceleration response? Clin Neurophysiol 2008;119:2362-9.

10. Minor LB, Solomon D, Zinreich JS, Zee DS. Sound- and/or pressure-induced vertigo due to bone dehiscence of the superior semicircular canal. Arch Otolaryngol Head Neck Surg 1998;124: 249-58.

11. Pellacani G, Seidenari S. Variations in facial skin thickness and echogenicity with site and age. Acta Derm Venereol 1999;79:366-9.

12. Driscoll C, Bekessy A, Bui V, Fox D, Harvey M, Mackenzie D. Vestibular evoked myogenic potentials: clinical implications of a normative investigation. Aust New Zealand J Audiol 2007;29:98-112.

13. Funk JR, Rowson S, Daniel RW, Duma SM. Validation of concussion risk curves for collegiate football players derived from HITS data. Ann Biomed Eng 2012;40:79-89. 